\title{
An optimization model for evacuating people with disability in extreme disaster conditions: A case study
}

\author{
Sadoullah Ebrahimnejad $^{\mathrm{a}, *}$, Michelle Villeneuve ${ }^{\mathrm{b}}$, and Reza Tavakkoli-Moghaddam ${ }^{\mathrm{c}}$ \\ a Department of Industrial Engineering, Karaj Branch, Islamic Azad University, Karaj, Iran \\ ${ }^{\mathrm{b}}$ Centre for Disability Research and Policy, The University of Sydney, Australia \\ ${ }^{\mathrm{c}}$ School of Industrial Engineering, College of Engineering, University of Tehran, Tehran, Iran
}

\begin{abstract}
The increasing severity and frequency of disasters have posed major challenges for people. Amongst, the risks of fatalities and injuries of people with disabilities (PWDs) have significantly increased. The Sendai Framework for Disaster Risk Reduction (SFDRR) initiated a movement to create a "disability-accessible and inclusive environment", which highlighted the problems PWDs faced during disasters. One of the most important issues is to provide evacuation and accommodation according to the special needs of PWDs. In this study, a mixedinteger linear programming (MILP) model is proposed to pick up PWDs from different locations and transfer them to shelters. Throughout this research, diverse disabilities, heterogonous vehicles, compatibility types of disabilities and vehicles, multi-depot, and adept and amateur operators are considered to help evacuate PWDs. Also, 27 problems are solved to examine the efficacy of $(\mu+1)$ Evolutionary algorithm (EA) in large-scale problems. Subsequently, a real case study with 500 nodes (including pick-up, shelter, and depot nodes) is analyzed. The computational results illustrate that by adding small-sized (car) and mediumsized (van) vehicles to the current fleet, the time for tours traveled significantly reduces. Finally, a sensitivity analysis is conducted to prepare some managerial implications for crisis managers during the occurrence of disasters to help PWDs during evacuation.
\end{abstract}

Keywords: People with disabilities; Disasters; Evacuation model; Shelters; Transportation, $(\mu+1)$ Evolutionary algorithm (EA).

\section{Introduction}

The occurrence of natural disasters has doubled in the last two decades compared to previous periods. Among these natural disasters, major floods, earthquakes, fires, and hurricanes have been the most threatening to people. Natural disasters kill an average of 60,000 people per year with a range of casualties between 1 to 4 percent. The calamitous impacts of these disasters can also be felt like millions of jobs, infrastructures, and homes are lost or ruined during natural disasters. Statistics show that in 2019, there were 170 major floods, 32 earthquakes, and 13 fires that occurred around the world. One billion people or about $15 \%$ of the world's population, have some form of disability, and this population between 110 million and 190 million people experience severe disabilities. Some studies [1-5] warned about the occurrence of disasters and their consequences. Due to climate change and rising sea levels, disasters (e.g., floods) will occur in the future more frequently. The destruction caused by floods would disrupt the transportation and accommodation of people during evacuation. These effects would be

\footnotetext{
* Corresponding author.

E-mail address: ibrahimnejad@kiau.ac.ir
} 
devastating for vulnerable people, particularly people with disabilities(PWDs). Moreover, floods reduce the reliability and efficacy of transportation infrastructures and impede communication and accessibility between communities. We can observe the tragic consequences of floods from an example that occurred in the UK in 2007 where 5500 homes were destroyed, and 6000 people lost their jobs.

The experiences of hurricane Katrina in 2005 in the United States showed that a significant lack of planning and management leads to a catastrophic disaster. In the aftermath of Hurricane Katrina, some government officials did not have sufficient experience in evacuation orders before the event, so they did not take appropriate measures to evacuate. During natural disasters, elderly people who could not walk had to stay at home [6-10]. Also, PWDs were severely affected by the risks of natural disasters. For example, only 41 of the 102 disabled people living in Galle, Sri Lanka survived the December 2004 tsunami since most of them could not leave their homes or did not realize the need to evacuate in time. Also, reports show that none of the 700 people with polio who lived on one of India's Andaman Islands could survive the tsunami because they couldn't be evacuated. Vulnerable people in communities are not able to properly evacuate themselves during natural disasters and may need targeted service support to gain access to food, health care, and social support. According to the Sendai framework [11], PWDs must be allowed to participate in society equal to all citizens. Ignoring this principle in the event of natural disasters is not justified. In such situations, PWDs may face physical barriers and communication challenges that prevent them from reacting effectively in critical situations and deter them from using the facilities and assistance available to others. Based on the literature, transportation is a very important policy issue for PWDs [12- 15]. PWDs constantly describe how barriers to transportation affect their lives in important ways. Over the past two decades, the National Disability Organization (NOD) has conducted three surveys that have identified transportation issues as a major concern for PWDs. Also, they found that those who suffer from more severe disabilities describe transportation problems more seriously.

For example, after Hurricane Katrina in the United States, according to the Emergency Management Reform Act of 2006, local governments were obliged to be responsible for planning evacuation for all, including PWDs. Reports show that evacuation planning has a key role in planning and relief operations in natural disasters. The major benefits of a timely evacuation are that the number of fatalities in disasters substantially decreases. When comparing the number of fatalities in a Super-cyclone in 1999 and the Cyclone Phailin east coast of India in 2013 a massive reduction has occurred from 10000 to 30. (New York Times, 2013). These significant figures reveal the importance of evacuation planning and relief operations. When evacuating PWDs, local authorities face many constraints, including the variety of disabilities, a limited number of vehicles to evacuate, and the different levels of functional mobility of evacuees. Also, all evacuations must be carried out within a limited time before or during a disaster. Another challenge is to minimize casualties in an emergency, and this often depends on the fast and efficient allocation of vehicles and routes. In the experience of hurricane Katrina, there were no plans to evacuate the dependents on transportation, and people with disabilities and chronic conditions did not receive good services.

Litman [16] reported that due to the lack of a comprehensive understanding of the decisionmakers with the various support needs of PWDs, effective natural disaster management and planning of evacuation operations for PWDs is of particular importance. The importance of 
evacuation planning with mathematical models after Hurricane Katrina and receiving reports became essential. Reports from hurricane Katrina showed that there was an unnecessary delay in the evacuation process due to personal vehicles causing congestion on various sections of the road. Malpass et al. [17] reported that natural disasters have affected the lives of more than 1.5 billion people. Due to the high vulnerability of PWDs, the risk of mortalities and injuries would be twice the risk of people without disabilities during natural disasters. Mace et al. [18] indicated that during natural disasters the multifaceted needs of PWDs create a significant risk to their lives. They demonstrated aspects of communication, medical needs, independence, supervision, and transportation (C-MIST). These aspects emphasize the support needs of PWDs in five areas including communication (C), medication and equipment (M), independence in performing daily activities (I), supervision (S), and transportation during evacuation to shelters to PWDs.

These numerous challenges during evacuation and transportation of PWDs include the variety and severity of disabilities, health conditions of PWDs, their special needs, successful communication and interactions between operators and PWDs, helping PWDs deal with the stress of leaving home in the absence of families and their caregivers, and public facilities are not equipped with the appropriate tools to provide easy accessibility for PWDs, lack of awareness of PWDs to their surrounding environment and recently occurring events. As an example, the COVID-19 pandemic revealed the risks to PWDs, especially those who had limited access to jobs, food, healthcare, and COVID-19 testing. These challenges have become more relevant with the recent COVID-19 pandemic. It is crucial to understand how PWDs are impacted in terms of health, transport, and education. This pandemic demonstrated the extreme difficulty in accommodating, safely managing, and communicating information about the disease to PWDs. Information about the symptoms and ways of prevention of COVID-19 was not provided to PWDs in accessible formats that cater to their specific disabilities, including Braille, audio provisions, and sign language interpretation. As a result, the number of fatalities of PWDs was immensely higher compared to ordinary people during this pandemic. The Office for National Statistics released an article highlighting COVID-19 related deaths from March to 14 July 2020 by disability status in England and Wales. Astoundingly, PWDs made up 59\% of deaths involving COVID-19 whereas they constitute just $22 \%$ of the whole population [see 19].

Wong et al. [20] reported a real-life case related to recent wildfires in the western United States (U.S.) and multiple hurricanes in the Gulf Coast simultaneously occurring with the COVID-19 pandemic which massively complicated the situations for stakeholders and services providers as well as local and state governments. This is because the orders and restrictions which were in place for the wildfires and hurricanes conflicted with health recommendations for COVID-19. PWDs experienced the (COVID-19) pandemic alongside extreme disasters. Moreover, the situation was exacerbated for PWDs due to the difficulty in communicating this complex situation to PWDs. Evacuation orders recommend people to leave their homes whilst the COVID-19 restrictions recommend people to stay home. In this conflicted situation, researchers addressed COVID-19 as a part of the evacuation planning and provided new protocols that followed COVID-19 restrictions whilst evacuating people. These protocols underlined the use of private vehicles to decrease spread COVID -19, whereas most PWDs did not have either a private vehicle or not able to drive. In addition to, transportation issues, assigning vehicles with high-capacity and congregate shelters, and resource sharing in shelters 
were other issues in this situation. Simultaneous occurrences of these disastrous events can have managerial implications for future evacuation planning. To help to decrease transportation problems, in this research, the aim is the optimal allocation of types of disabilities to heterogeneous vehicles and vehicle routing to pick up PWDs during evacuation operations. We propose an MILP model for evacuating PWDs to shelters, before the occurrence of natural disasters. The operation to evacuate and transfer PWDs to shelters encounters many challenges. Based on previous studies, gathering the challenges of PWDs and vulnerabilities during natural disasters can be examined from several aspects, most of which are mentioned below.

- Separation of vulnerable and disabled groups, in terms of their evacuation and transfer conditions (the problems separating children from families, the elderly, do not separate the mentally and physically disabled from their caregivers, how to transfer pregnant women)

- How to transfer PWDs according to their type of disability

- Accessibility problems to special purpose vehicles for transporting PWDs and the limited number of vehicles

- Different challenges in dealing with the type and severity of natural disasters and their appropriate evacuation procedures

- Problems transporting PWDs to the shelter (such as traffic on affected areas - duration on the way due to their disability and fatigue - First aid along the way and the need for medical equipment along the way)

- Refusal of help from some members of the rescue team (especially the mentally handicapped or mentally ill)

- Lack of necessary training for the disabled people, caregivers, and the rescue team itself

- Problems related to lack of sufficient information about PWDs (their location, medical history, and medical needs at the time of transfer and necessary medications)

- Problems with how to communicate with PWDs during their evacuation and transfer, and in some cases insufficient information to contact the families, caregivers, and friends of PWDs.

- Lack of trained personnel, nurses, volunteers in times of crisis

- Problems of proper distribution of shelters in terms of location for better access and having infrastructure facilities and lack of crowded shelters for proper access to resources and to prevent the spread of infections such as COVID-19

- Serious challenges for PWDs with multiple acute illnesses such as:

- Chronic diseases, especially heart disease, cancer, stroke, diabetes, and chronic respiratory disorders and require special care at the time of transfer

- Lack of transparency in the services of some governmental and non-governmental organizations to assist PWDs in the event of natural disasters

- Multiplicity of stakeholders in the evacuation plan

- Lack of integrated documentation of events during the transition in previous crises to analyze them for the upcoming crisis planning

- Problems with the early warning system, especially for the disabled, such as the deaf and dumb, to alert them before a crisis occurs 


\section{Literature review}

Detti et al. [21] suggested a multi depot dial-a-ride problem (DARP) in the healthcare sector for transporting non-emergency patients with a real-world application. They took into account some constraints such as heterogeneous vehicles, patient compatible vehicles, service quality requirements, patients' preferences, and the cost of used vehicles. Transportation services and the types of vehicles are two additional fundamentals elements considered in this research. Parragh et al. [22] presented a model that proposes to minimize total routing costs considering upper bound route duration, time windows, and upper bound user ride time. In this paper, they proposed a variable neighborhood search (VNS) algorithm for the DARP. Melachrinoudis et al. [23] formulated an MILP mathematical model for a static DARP with soft time windows and solved it using a metaheuristic algorithm. The aim was to investigate a dial-a-ride system that serves outpatients such as drug addicts and alcohol addicts at the Center of Addictive Behavior Health and Recovery Services Inc. (CAB), a non-profit organization in Boston. The proposed objective function considers total cost and total client waiting time which is comprised of vehicle riding time and the amount of time delivery services violated time windows. Parragh et al. [24] proposed models in static DARPs for patients' transportation mainly used by the Austrian Red Cross (ARC). Models and algorithms are designed for a static DARP aiming to minimize the cost of routing. The problem is described as a heterogeneous DARP with driver restrictions (HDARPD).

Lehuede et al. [25] introduced a multi-criteria model from multi-attribute utility theory using the Choquet integral and modeled it as a DARP, addressing transportation of PWDs from home to special centers or schools. Mentally disabled patients' transportation was considered in this research. They emphasized that due to a lack of autonomy, these patients cannot use public transportation. For this reason, specialized transportation services were utilized, including eight-seater minibusses. Three main applications included in this research were the transportation of adults to vocational rehabilitation centers, the transportation of disabled children to specialized schools, and the transportation of disabled children to regular schools.

Rais et al. [26] presented a pick-up and delivery problem with and without time windows for services and presented mixed integer programming(MIP) models to solve these problems. They considered further generalization by allowing transshipment in the network and they also considered heterogeneous vehicles. This study aimed to minimize vehicles routes costs for meeting all customer demands. Feng et al. [27] presented an MIP model and a heuristic approach for solving real-world and large-scale problems. The objective function considers the sum of fixed and operational costs. An evacuation scenario was generated based on the realworld evacuation zones, shelter locations, and population statistics for New York City. Faria et al. [28] presented a model in the DARP to transport PWDs, aiming to improve their lifestyle and provide better service. They proposed a parallel insertion heuristic in a real-life DARP in a medium-sized city.

Melachrinoudis [29] investigated a case study in a center for addictive behavior health and recovery services Inc. (CAB) in Boston. A DARP was designed to serve alcohol and drug addicts and service them via telephone calls. As the number of these patients is increasing, $C A B$ copes with these tasks by minimizing the service disruptions and total costs of vehicles. To cope with this challenge, they developed an MILP model and a tabu search method to deal with 
a DARP considering one-sided soft time windows. Reinhardt et al. [30] reported that many airports around the world use special kinds of transportation for passengers with reduced mobility. The time window between pick-up and getting on board is too short and, in this time, some passengers with reduced mobility (PRMs) might be left. As PRMs have difficulties in movement, it is important to develop transportation programming. The problem aims to plan passengers' travel while ensuring smooth transport with a short waiting time. Parragh [31] introduced a mathematical model, considering a DARP deals with transporting people from a pick-up center and delivering them to a predetermined location. The objective of this problem is determined by minimizing the routing costs, serving all patients and PWDs, and reducing their waiting time. In this problem, restrictions associated with the quality of service are defined by time windows and an upper limit for user ride time. Four different transportation modes are presented in this model including patient seats, wheelchair places, staff seats, and stretchers. Riedler and Raidl [32] considered a DARP includes transportation requests from pick-up to drop-off points. All the requests are served by limited capacity vehicles and tour duration per vehicle. Also, it is presumed that by limiting the travel time between origin and destination for requests, user inconvenience considerations. In this paper, different scenarios are considered, and the maximal number of requests should serve under different constraints but also there is no guarantee for serving all of the requests. A compact MILP model and algorithms are introduced based on Benders decomposition.

Cordeau and Laporte [33] presented a tabu search method for static DARPs considering multi-vehicle with predetermined pick-up and delivery nodes, different time windows, and depots. They proposed to formulate a set of minimum-cost vehicle routes that can serve all demands. Route duration, vehicle capacity, and the maximum ride time of any user are some of the restrictions. Parragh et al. [34], discussed challenges the Austrian Red Cross (ARC) faced as an ambulance dispatcher. Today ARC, as a nonprofit organization, is forced to reduce its operating costs and also consider minimizing costumers' inconvenience and maximum riding time constraints. Molenbruch et al. [35] proposed a bi-objective DARP, considering real-life characteristics and problems faced during patient transportation. A multi-directional local search algorithm is provided to solve this problem, taking service quality and operational efficiency into account. The problem is presented with combination restrictions, usually caused by medical reasons. This paper emphasizes the conflicting interests of service providers and users in the objective function in a DARP model.

Cheng [36] created a dial-a-ride service system for elderly people based on their location. This study established a location-based service system, which monitors the position of vehicles and responds quickly to user requests. By using a mobile phone, a user can send a request to the operation center. Users' is location automatically detected and location information is sent to the operations center along with the delivery time and destination requested. After that, routing and dispatching of the vehicles are processed by a module. If feasible, one of the vehicles rerouted and a user will be picked up from a certain location. In this study, the rerouting and dispatching of a vehicle to respond to a user's request are formulated as a DVRP.

Tellez et al. [37] presented a DARP with heterogeneous users, where the capacity of the vehicle on the route is reconfigurable and can be changed by resetting the internal arrangement of the vehicle. The proposed dial a ride problem with reconfigurable capacity (DARP-RC) is an extended problem of the classic DARP with more realistic assumptions, such as 
heterogeneous users (e.g., seats, wheelchair, and trolleys) and reconfigurable vehicles. The objective function considers the total transportation costs. Given the number of passengers and vehicles in large-sized problems, MIP solvers and exact solution methods are not able to solve these problems. They used a neighborhood search meta-heuristic integrated with a set covering module. Ropke and Pisinker [38] proposed a pick-up and delivery problem assessing time windows. They developed a heuristic problem based on a large neighborhood search heuristic. In this case, the objective function minimizes the total weighted mileage, the total time spent by each vehicle, and the number of unscheduled requests arrived.

Kaisar et al. [39] developed a linear programming optimization model to determine the optimum location for evacuation centers. They used a microscopic traffic simulation model. The main objective of their study was to evaluate different evacuation procedures and plans for PWDs using public transportations. Input data in their model includes geometric design, signal timing, traffic demand, and demographics. Dhamala and Adhikari [40] presented a holistic overview of the evacuation planning optimization methods which have been developed during recent years. They reviewed their formulating model and its solutions about disaster management from the transportation perspective. Numerous mathematical models and algorithms have been formulated during several years to solve transportation-based evacuation under both predictable and unpredictable disasters. Murray-Tuite and Wolshon [41] reviewed the modeling and simulation of highway-based evacuation. They surveyed the studies conducted over the past decade. Their review also includes the main components of road transport operations planning.

Bian and Wilmont [42] estimated the spatial distribution of the population using a modified dasymetric mapping method, they considered population distribution inland parcels with dimensions $30 \mathrm{~m}$ by $30 \mathrm{~m}$. In each parcel, the vulnerable population is estimated with regards to the proportion of disability, age, and zero car-owning persons in the area based on census data. The results of this study showed that demographic information is beneficial in identifying areas in a metropolitan region where transportation services are needed during evacuation. They used an optimization model to determine the pick-up points to serve the vulnerable population in the metropolitan areas. Bayram [43] presented a comprehensive literature review in evacuation planning based on traffic assignments under static and dynamic conditions. They also presented studies that propose effective supply and demand management strategies. The goal of these studies is to achieve evacuation operations to prevent congestion. Amideo et al. [44] examined the research gap related to shelter location and evacuation routing operations. In their research, they described issues such as stakeholder engagement, evacuation modes, clear definition of parameters and inputs of optimization models, the behavior of evacuees, and system behavior in nine case studies in detail. Finally, they presented their research findings in the form of future research, the main points of which are as follows:

Use of research approaches in soft operations research / problem structuring methods (OR / PSMs) operations, disruptions modeling in infrastructure, development of stochastic models taking into account several objectives and several periods, and emphasis on collecting primary data at the level of stakeholders such as evacuees. Shahparvari and Abbasi [45] proposed a stochastic MILP model for emergency fire evacuation. They considered the required number of vehicles, evacuation schedule, uncertain access of routes in population evacuation, and limiting the maximum pre-determined evacuation time. They developed the greedy solution 
method for their proposed model and compared it with a designed genetic algorithm for the effectiveness of their solution method. Dulebenets et al. [46] investigated the issue of largescale emergency evacuation in the event of natural disasters. They studied decisions about evacuation routes, emergency shelters, evacuation periods. They presented a multi-objective mathematical model in which the main driver characteristics, evacuation route characteristics, driving conditions, and traffic characteristics of the evacuation route were considered. Baou et al. [47] proposed an MILP model for evacuating PWDs in an emergency. One of the features of their model is the use of two types of heterogeneous vehicles (especially for the disabled) and ambulances to transport the mobility-disabled people to shelters. They also considered the time window limit for the pick-up points. Also, they proposed a fast-innovative solution method to solve their model that could be compared to an exact solution method to determine the efficiency of innovative solutions.

Flores et al. [48] proposed a Lexico goal programming model for supported evacuation to rescue people in natural disasters. They proposed a fast-innovative solution method to solve their model that could be compared to an exact solution method to determine the efficiency of innovative solutions. They defined the attributes of the number of people with high priority for evacuation, the number of people with normal priority, the total evacuation time, and the operating cost in the first to fourth order in the objective function. They also considered the time limit for transferring high-priority people before normal people in their proposed model. Zahao et al. [49] proposed a two-objective mathematical model in which the first goal is to minimize the shelter area and the second goal is the evacuation time. Their proposed model examines the relationship between evacuating population size, shelter capacity, and evacuation time. They also consider limiting the distance of evacuees from the maximum distance set for the location of shelters. Bolia [50] proposed an MILP model and determined the operational strategy of buses for evacuation. They also obtained the minimum number of buses to evacuate the affected area in their proposed model.

Swamy et al. [51] studied the evacuation of people before the occurrence of a hurricane on a large scale. The evacuation strategy in large volumes was using public transportation. They used a multi-step approach to their proposed evacuation model. First, they identified the locations of the pick-up and the shelters. They then produced a series of routes leading to the shelters along with the pick-up sites. They developed a simulation tool to consider the number of buses being sent, possible entry of evacuees to shelters. Dulebenets et al. [52] proposed an MILP model that assigns vulnerable population groups to emergency shelters through the evacuation route during available periods. They developed a simulation tool to consider the number of buses being sent, possible entry of evacuees to shelters. The proposed objective function is to minimize the total travel time of people in the evacuation area. Among the features of their proposed model are considering the social-demographic characteristics of the evacuees, the characteristics of the evacuation route, driving conditions, and traffic characteristics about the evacuation time of the evacuees. To solve the proposed model, they proposed four scenarios for the innovative algorithm and compared the results with the exact method, which confirms the efficiency of their innovative algorithm. Gao et al. [53] proposed a robust two-stage model for evacuation in response to large-scale natural disasters. In the first step, they developed a nonlinear integer programming model that determines the pick-up, routing, and scheduling locations. Secondly, they proposed an MILP model that allocates vehicles to the pick-up points 
of evacuees to shelters. The goal is to minimize evacuation time. They used a hybrid genetic algorithm to solve their model.

In this study, an MILP model has been proposed to evacuate PWDs before a disaster occurring (in warning time). It considers key elements in an evacuation operation. These elements are different disabilities, heterogeneous vehicles, and recruiting operators in two skill levels (the trained operators and ordinary operators). These operators play a key role in better communication and cooperation with PWDs in requested locations to be prompt and efficient during evacuation. Also, the type of objective function is defined as a min-max form, which minimizes the maximum longest tour in an evacuation network. Also, a $(\mu+1)$ Evolutionary algorithm (EA) has been designed to solve the proposed model. Finally, the proposed model was implemented in a case study in Kermanshah city (Bakhtaran). Three sensitivity analyses were conducted to assess the reduction of evacuation time considering the impact of adding a certain type of vehicle in the current fleet, adding a backup shelter to equipped shelters (active shelters) that are ready to serve and, using two different objective functions, either minimizing the total time traveled or minimizing the maximum time of each tour to determine which objective function uses the least number of vehicles. The paper is organized as follows. In Section 2, a literature review is described. In Section 3, the problem description and an MILP model are respectively presented. In Section 4, we provide the computational results using an exact method (CPLEX solver) and ( $\mu+1)$ EA and an analysis of a real-life case. In Section 5, we present a conclusion and future research.

\section{Problem description}

This study emphasizes on emergency evacuation of PWDs to shelters when a disaster triggered by natural hazard emergencies occurs. One of the key elements in transportation is the level of experience and knowledge of service providers when helping PWDs. Moreover, access to a real emergency action plan and adequate human resources, special purpose vehicles for PWDs, access to road networks in emergencies, and using air transportation are all crucial elements when developing an evacuation plan for PWDs. Therefore, the variety of needs and the complexity of unpredictable events jeopardize PWDs and service providers during evacuation operations. Learning and transferring necessary knowledge from experiences of previous disasters can greatly help service providers and other operators during evacuation operations. It is necessary to identify the special needs of PWDs during their transportation. As a result, the executive procedures for transferring PWDs must be based on the type of disability. In this problem, we consider several types of disabilities, vehicle compatibility with the types of disability, vehicle capacity, a displacement map of PWDs, shelter points, and distances between the pick-up points and between the pick-up and delivery points are predetermined. It is also assumed that operators are assigned to vehicles based on their experiences about the type of disability and familiarity with the particular disability. The types of disabilities include sight and hearing, mobility disability, mental illness, and mental disorders, and combined mobility and mental disability. This study aims to minimize evacuation time. To achieve this objective, maximum vehicle tours in collecting and transporting PWDs to shelters are minimized. By assigning vehicles to the tours, this objective minimizes the time of collecting and transferring PWDs. It should be noted that there are enough vehicles considered for the pick-up and delivery operations. In this study, PWDs are divided into four groups in the evacuation plan: 
- The first group (G1) includes people with visual or hearing impairments or communication disabilities.

- The second group (G2) includes people with mobility impairments who often use equipment such as wheelchairs or mobility scooters.

- The third group (G3) includes people with mental illnesses and mental disorders.

- The fourth group (G4) includes people who have both mobility and mental illnesses.

At the time of natural disasters, due to stress and anxiety, several PWDs, especially those with mental illness, may show unexpected behaviors and reactions. For this reason, it is necessary for operators, who assist these people in evacuation to have passed the necessary training and be skilled enough to face the challenges during evacuation time. Therefore, two groups of operators are defined. The first group is operators who do not need to undergo special training. These operators can handle disabilities that do not pose many difficulties in terms of communication and collaboration with PWDs (e.g., group G1). The second group is operators who need to have the necessary training to be able to communicate and help people with special disabilities (e.g., mental illness) during the time of evacuation and transportation. Vehicles are categorized into three types that have small, medium, and large capacities. Small vehicles have a capacity of four people, medium vehicles have a capacity of 8 to 10 people and large vehicles have a capacity of 20 people. It should be noted that medium-capacity vehicles are equipped so that they can serve all of the four disability groups, especially those who have mobility disabilities. For example, in Fig. 1, three paratransit vehicles with different configurations are displayed. Another type of vehicle is used bus and SUV cars (www.freedomaccessvehicles.com.au/vans-buses/mercedes-sprinter.php[54]).

\{Please insert Fig. 1 about here. $\}$

According to the aforementioned two groups of operators and three types of vehicles, six modes are considered separately, which are:

1. Bus type vehicle with a trained operator.

2. Bus type vehicle with an ordinary operator.

3. Paratransit vehicle with a trained operator.

4. Paratransit vehicle with an ordinary operator.

5. Car with a trained operator.

6. Car with an ordinary operator.

Now, these four disability groups are assigned to six modes. Group G1, includes PWDs who have visual and auditory disabilities, and they are assigned to any type of vehicle with any type of operator. Group G2 is PWDs with mobility disabilities and is allocated to paratransit vehicles with any operator. Group G3 includes PWDs with mental illnesses is assigned to any type of vehicle; however, the operators who help in evacuating this group must be trained. Group G4 includes PWDs who have a combination of mental and mobility disabilities assigned to paratransit vehicles with trained operators.

In this study, a set of requests related to PWDs is considered and the aim is to transfer them from their position to one of the pre-determined shelters before the occurrence of natural disasters. For this purpose, $P=\{1, \ldots, n\}$ is defined as a set of locations of PWDs, 
$D=\{n+1, \ldots n+s\}$ is defined as a set of locations of shelters, and $K=\left(m_{1}, m_{2}, \ldots, m_{k}\right)$, is a heterogeneous set of vehicles where $m_{k}$ is the number of vehicles of type $k$. Due to the different types of disabilities and the particular compatibility of vehicles to these disabilities, certain disability groups must be allocated to certain vehicle types. For this purpose, a matrix called $f(p, k)$ was considered and if vehicle type $k$ can serve the disabled person located in place $p$, the value is 1 , otherwise 0 . The matrix $b(p, g)$ shows that if the disabled person located in place $p$ has disability $g$, the value is 1 and otherwise 0 . The matrix $c(k, g)$ indicates that if the disability $g$ can use the vehicle type $k$, the value is 1 , otherwise 0 . Therefore, the value of $f(p, k)$ is obtained based on $\sum_{g} b(p, g) * c(k, g)$ Given the relation between the types of disabilities and compatibility of vehicles to these disabilities, and the requested service in the pick-up nodes, compatible vehicles are departed to demand nodes.

It is assumed that each vehicle starts its tour from depot $r \in O$ and is sent to one of the shelters after collecting PWDs who are compatible with the type of vehicle and do not exceed the capacity of the vehicle. Thus, for each pick-up node, only one vehicle can visit. For each request $i, p(i) \in P$ andets Sup and delivery points, respectively. -the pickre $d(i) \in D$ . and depots up and delivery points-are all pick $V=\{1,2, \ldots, n+s+r\}$ and $N=\{1,2, \ldots, n+s\}$ Each trip from location $i \in V \backslash D$ to location $j \in V \backslash P$ is made at a distance of $d_{i j}$. In the occurrence of natural disasters, evacuation time plays a key role in reducing risks for PWDs. By traversing to PWD pick-up nodes according to the proposed model, each vehicle creates a tour that minimizes the evacuation time and so the risk of PWDs being trapped during the evacuation operation decreases. This objective function minimizes the maximum tour times traveled by vehicles to pick up PWDs in the affected area. Besides, it balances the time traveled by each vehicle based on its capacity in each tour and enables each vehicle to travel a tour in the shortest time possible. Hence a min-max objective function is suitable for this problem.

\subsection{Model formulation}

Model assumptions:

1. The locations of PWDs are already known. (predetermined)

2. The types of vehicles are already known.

3. The types of disabilities are already known.

4. There is no priority for picking up PWDs.

5. The number of PWDs in need of evacuation is known.

6. The number of vehicles of any type is known.

7. Travel time between two pick-up and delivery points or two pick-up points is deterministic.

8. During the pick-up and drop-off nodes of PWDs, attendants are not picked up.

9. The conditions of the shelters are the same.

10. Each disabled person will be picked up from a given pick-up point.

11. Each vehicle traverses one tour. 
12. The location of vehicles for the transport of disabled people across the city is predetermined.

13. The location shelters are known.

14. The severity of a natural disaster and its type (storm, flood, tornado, and fire) are predictable.

15. The number of trained/untrained operators is already known.

\section{Indices:}

$$
\begin{array}{lr}
\begin{array}{ll}
i, j & \text { Consecutive the pick-up points or pick-up and delivery points } \\
m_{k} & \text { Number of vehicles type } k
\end{array} \\
\begin{array}{l}
M \\
N_{1}=\{1,2, \ldots ., n\} \in P \\
\text { Type of disability }
\end{array} \\
N_{2}=\{n+1, \ldots, n+s\} \in D \\
O=\{n+s+1, \ldots ., n+s+r\} \\
N=\left\{N_{1} \cup N_{2} \cup O\right\}
\end{array}
$$

\section{Parameters:}

$Q_{(k)}$

$b_{(i, g)}$

$f_{(j, k)}$

$C_{(k, g)}$

$d_{(i, j)}$

$V_{(K)}$

$h_{(r, k)}$ If the vehicle type $k$ belongs to depot $r$, it is equal to 1 and otherwise 0

$t_{(i, j, k)}$

$K=U_{l=1}^{k} m_{l}$

Set of all vehicles from all available types

$t_{(i, j, k)}=d_{(i, j)} / V_{(k)}$

$f_{(p, k)}=\sum_{g} b_{(p, g)} \times c_{(k, g)}$

$x_{i j k}$

If $\operatorname{arc}(i, j)$ is traversed by vehicle type $k$, it is equal to 1 and otherwise 0

$\sum_{j \in P \cup D} \sum_{k} x_{i j k}=1$ 


$$
\begin{aligned}
& \sum_{i \in O \cup P} x_{i j k}-\sum_{i \in P \cup D} x_{j i k}=0 \quad \forall j \in P, \forall k \in m_{k} \\
& \sum_{i \in P} x_{i j k}-\sum_{i \in O} x_{j i k}=0 \quad \forall j \in D, \forall k \in m_{k} \\
& \sum_{i \in O} \sum_{j \in P} x_{i j k}-\sum_{i \in P} \sum_{j \in D} x_{i j k}=0 \quad \forall k \in m_{k} \\
& \sum_{i \in P} \sum_{j \in D} x_{i j k} \leq 1 \quad \forall k \in m_{k} \\
& \sum_{j \in P} x_{i j k} \leq h(i, k) \quad \forall i \in O, \forall k \in m_{k} \\
& M\left(\sum_{i \in O} \sum_{j \in p} x_{i j k}\right) \geq \sum_{i \in P} \sum_{j \in P \cup D} x_{i j k} \quad \forall k \in m_{k} \\
& \sum_{i \in O \cup P} \sum_{j \in P} x_{i j k} \leq Q_{(k)} \quad \forall k \in m \\
& \sum_{i \in O \cup P} x_{i j k} \leq f(j, k) \quad \forall j \in P, \forall k \in m_{k} \\
& u_{i}-u_{j}+|n| x_{i j k} \leq|n|-1 \quad \forall i, j, k \\
& x_{i j k} \in\{0,1\}, u_{i} \geq 0 \quad \forall i, j, k
\end{aligned}
$$

Equation (1) Minimizes the maximum tour time between the pick-up and drop-off points for customers (disabled). Constraint (2) ensures that only one type of vehicle exits at each delivery point. Constraint (3) ensures that any vehicle entering any node leaves the same node. Constraint (4) ensures that every vehicle from any pickup node to any delivery node finally returns to the depot node. Constraint (5) guarantees that any vehicle entering one of the pickup points will be delivered to one of the delivery points. Constraint (6) ensures each vehicle can enter from one of the pickup nodes to one of the delivery nodes. Constraint (7) indicates that vehicle $k$ can depart from depot $r$ to one of the pick-up points. Constraint (8) ensures that vehicle $k$ only can travel between nodes, only if it is sent from depot $r$. Constraint (9) shows the capacity of vehicle $k$. Constraint (10) ensures that vehicle $k$ is only sent to pick up node $j$ if it is compatible with the type of disability in node j. Constraints (11) express sub-tours restrictions. Constraint (12) shows the binary variables and Auxiliary variables. Given that Equation (1) is a nonlinear relation, it can be converted to linear form as follows:

$$
\max _{k} \sum_{i} \sum_{j} t(i, j, k)^{*} x_{i j k}=Z \quad \forall k \in m_{k}
$$


As a result, the linearized model is presented by:

(P2): $\operatorname{Min} Z$

Constraints (2) $-(12)$

\subsection{Solution approaches}

The proposed MILP model consists of $|N|^{2} \times K+N-1$ decision variables and $|N(N-1)| \times|K|+|N 1| \times|(2 K+1)|+|k| \mid(|N 2|+|O|+5 \mid)+1$ constraints. This model can be solved small and medium-sized problems by the branch-and-bound method, which are solved using GAMS software and CPLEX solver by a laptop with $8 \mathrm{G}$ of ram and Core i5 CPU.

The proposed mathematical model is very similar to the dial a ride problem in terms of the constraints; however, in the proposed model a vehicle picks up all of its demand nodes and delivers them to one shelter. The difference in the evacuation problem is that initially, all the demand nodes are picked up by vehicles and after a vehicle picks up all of its demand nodes it is sent to one of the shelters. Also, in the proposed mathematical model, the sub-tour constraints are taken into account. Therefore, the constraints of the proposed mathematical model are similar to the constraints of the classic DARP and the traveling salesman problem. Both TSP and DARP are NP-hard models. Thus, the proposed model also failed to produce feasible/optimal solutions by solving medium to large size problems at reasonable times (up to 2 hours). For example, for problems larger than $P=150$ customers, $D=7$ shelters, $K=16$ vehicles, and $O=3$, the CPLEX solver could not solve the problem in two hours. Hence to solve these large-scale problems, we must use meta-heuristic algorithms. For this purpose, in this research, the $(\mu+1) \mathrm{EA}$ will be used to solve large-scale problems.

\section{3. $(\mu+1) \mathrm{EA}$}

In this section, we present an EA capable of solving large instances of the problem. The algorithm is initialized with $\mu$ random solutions. In the second stage, a solution $I$ is chosen randomly, and an offspring $I^{\prime}$ is generated by mutation. Next, the offspring is added to the population, and an individual with the worst objective function is discarded from the population. Having had $k$ consecutive iterations without improving the best objective function within the population, the restart mechanism is implemented. These steps are repeated until a termination criterion is met. Throughout this study, the algorithm is terminated when five consecutive restart mechanisms take place without improving the best-found solution. Algorithm 1 represents the proposed algorithm. 


Algorithm $1(\mu+1) \mathrm{EA}$
1: Initialize the population $P$ with $\mu$ random solution
2: Choose $I \in P$ uniformly at random and produce an offspring $I^{\prime}$ of $I$ by
the mutations.
3: Remove exactly one individual $I$, where $I$ has the worst objective
function value
4: If there is no improvement in the best-found solution objective value
for $k$ consecutive iterations, implement the restart mechanism.
5: Repeat Steps 2 to 6 until a termination criterion is reached.

A chromosome structure is represented as a solution according to Fig. 2. In this string, there are m vehicles that are separated by " 0 ". Therefore, each vehicle shows a tour. Locations $D, P$, $S$ indicate depot, pick-up, and shelter nodes, respectively.

\{Please insert Fig. 2 about here.

\section{- Restart Mechanism}

In multi-module problems, EAs generally have immature convergence to local optimums. Routing problems (e.g., TSP and VRP) are widely used. The problem under investigation is not different from those routing problems. In this study, we use a mechanism to avoid immature convergence. In this mechanism, we replace the better half of a population with random solutions after a specific number of consecutive iterations without improvement such that the algorithm gains a chance to converge to better solutions.

\section{- Mutation}

The proposed mutation is based on 2-OPT, which is well-known and a powerful neighborhood search. However, two additional steps are added to 2-OPT to tailor the mutation for the problem. These steps boost the quality of mutants as well as the probability of generating feasible offspring. In the general 2-OPT, two elements within the array are selected and swapped randomly. Subsequently, every element in the sequence between these two selected points is replaced in reverse order. However, it can easily make an infeasible solution since shelters are required to be the last place visited before the depots. Thus, we discard the elements associating with shelters and depots before the implementation of 2-OPT. Having implemented the mutation, we have a sequence of visiting the pick-up nodes for each tour. Therefore, in each tour, we assign the last visited pick-up point to the closest shelter. Fig. 3 present the mutation operator.

\{Please insert Fig. 3 about here.\} 


\section{Computational results}

In this section, 27 instances were designed and solved. The dimensions of these instances are determined by the number of demand points, shelter points, and the number of vehicles and depot nodes. The instance with the smallest dimension had 15 demand points, 3 vehicles, 2 shelters, and one depot and while the largest instance consisted of 150 demand points, 16 vehicles with different capacities, 6 shelter points, and 3 depots. For 27 designed instances, the distance between points is generated randomly using a uniform distribution of $U(30,50) \mathrm{km}$ and with a normal distribution of $\mathrm{N}(10,3)$ and $\mathrm{N}(50,3) \mathrm{km}$. Also, the speed of small vehicles is 2 times faster than larger vehicles and 1.5 times faster than average-sized vehicles. The travel time between two nodes is equal to the generated distance divided by the speed of the traveling vehicle. Also, the distances between points $(i, j)$ and $(j, i)$ are considered equal (symmetrical distances) and the distances between points are assumed to be rectangular. Also, Table 1 shows the allocation of the four disability groups to the six modes in evacuation operations. It should be noted different modes cannot assign to different types of disabilities. For example, mode one is not compatible with disabilities G2 and G4.

\{Please insert Table 1 about here.\}

\section{- Solution approaches}

Due to the limitations of the CPLEX solver in solving large-scale problems, the $(\mu+1)$ EA was designed to solve large-scale problems with higher quality and low CPU time. The designed algorithm has great accuracy in solving small and medium scale problems, for example, in the instance with dimensions $(50 \times 4 \times 6 \times 2)$, the difference between the objective function value in the meta-heuristic method and the exact method is only $1 \%$. By solving a large number of instances with small and medium dimensions confirm this accuracy. For the problem with dimensions $(75 \times 4 \times 8 \times 2)$ and greater, the exact method was not able to find a feasible solution. The algorithm $(\mu+1)$ EA was able to find feasible solutions with a low standard deviation and is expected to generate near-optimal solutions based on the results of the small and medium-sized problems. Therefore, it can be expected that this algorithm can produce solutions with acceptable accuracy for larger problems. Thus, it should be noted that the CPU time of the $(\mu+1) \mathrm{EA}$ is substantially less than the exact method, and for problems with dimensions larger than 100 nodes, the exact method cannot even find a solution in a reasonable amount of time. The proposed model was solved using GAMS software with CPLEX solver and $(\mu+1)$ EA for 27 instances in which its results have been reported in Table 2.

\{Please insert Table 2 about here.\}

As shown in Table 2, three sets of 9 instances are generated by variation in distances and the number of vehicles in each mentioned vehicle type. The distances in the first set of 9 instances are produced randomly using uniform distribution $U(30,50)$. Within this first set, the CPLEX solver generated a feasible solution for the problem with dimension $75 \times 4 \times 8 \times 2$ whilst the $(\mu+1)$ EA algorithm obtained a solution that had a lower evacuation time by $11 \%$. In terms of CPU time, the CPLEX solver took 7200 seconds to solve the before-mentioned problem, while the 
$(\mu+1)$ EA only took 345 seconds. The distances are randomly generated in the second set of 9 instances by using a normal distribution $N(50,3)$. In the second set, the CPLEX solver found a feasible solution for the problem with dimension $90 \times 6 \times 10 \times 3$ whereas the $(\mu+1)$ EA found a solution that had a significantly lower evacuation time by $52 \%$. The CPU time of the CPLEX solver for this problem was 7200 seconds whilst it took $(\mu+1)$ EA 462.7 seconds. The distances in the third set of 9 instances are generated randomly by using normal distribution $N(10,3)$. In this third set, the CPLEX solver obtained a feasible solution for the problem with dimension $110 \times 5 \times 11 \times 3$ while $(\mu+1)$ EA could obtain a solution that improved the efficiency of the objective function by $52.5 \%$. In regards to CPU time, the CPLEX solver took 7200 seconds to solve the mentioned problem while $(\mu+1)$ EA only took 55.3 seconds. Through the comparison of these instances, it is evident that $(\mu+1)$ EA performs better compared to the CPLEX solver in terms of the quality of solutions and CPU time in large-scale problems. In this section, the obtained routes from both the exact method (CPLEX solver) and $(\mu+1)$ EA were presented for an instance with 25 pick-up nodes and four shelters.

According to an instance with 25 pick-up nodes, the maximum evacuation time among tours is 385 minutes. In this instance, it is assumed that the distance between nodes is generated randomly from a uniform distribution $U(30,50)$ and the speed ratio is equal to $X, 1.5 X$, and $2 X$ for bus, van, and car respectively. As shown in Table 3, the optimal solution generated by the CPLEX solver is the same as the best solution generated by the $(\mu+1)$ EA algorithm. Also, the standard deviation for ten runs generated by $(\mu+1) \mathrm{EA}$ is equal to 0.674 minutes, which is slightly low and this shows the accuracy of the algorithm.

As shown in Fig. 4, for the first four first instances CPU times are close to each other, but from instance 5 and onwards, there is a significant difference between CPU times of the exact method and $(\mu+1)$ EA algorithm. From instance 6 , the exact method can only find a feasible solution in only some instances and if the solving instance took more than 7200 seconds the solving time was truncated.

\{Please insert Table 3 about here.

\{Please insert Fig. 4 about here.\}

The original case consists of 66 vehicles and five shelters. In case 1, we added the sixth shelter. In the case of V1, we added a bus with a trained operator and decreased a bus without a trained operator in case V2. In the case of V3, we added two paratransit vehicles with tainted operators and decreased two paratransit vehicles without trained operators in case V4. In the case of V5, we added six SUV cars with trained operators and decreased six SUV without trained operators in case V6. We assumed each bus is equivalent to two paratransit vehicles and six SUV cars.

As shown in Table 4, according to Kruskal-Wallis statistical test, Bonferroni test, and using SAS 9.1 software, we concluded that the original case and case one are not significantly different at the $95 \%$ confidence level, which means adding a sixth shelter does not create an influencing decreasing in evacuation time (due to locating on the outskirts of the city). Also, there are significant differences between Case V2 and case V5 based on evacuation time at the 95\% confidence level. Besides, there are significant differences between Case V5 with the original case and case one based on evacuation time. Also, there are significant differences 
between Case V6 and the original case and case one based on evacuation time. The reason to be significant differences between cases V2 and V5 is that in the case of V2, are used buses without trained operators, whereas, in the case of V5, is used SUV cars with a trained operator which leads to decrease evacuation time. Also, a comparison between case V6 and the original case showed that in case V6, increasing the number of SUV cars decreases significantly evacuation time. Also, in Table 5, the results showed that there are significant differences between using a restart mechanism and not using a restart mechanism $(\mathrm{P}<0.05)$ so that the effect of the restart mechanism improves the quality of solutions. The implementation of the restart mechanism showed the range of improvement of solutions changes between $3 \%$ up to $45 \%$.

\{Please insert Table 4 about here.

\{Please insert Table 5 about here.\}

\subsection{Case study}

Iran is one of the most earthquake-prone countries in Asia, with 50 earthquakes in the last 100 years, killing at least 126,000 people. The magnitude of these earthquakes ranged from 5 to 7.7 on the Richter scale and most of them were above 6 Richter. Among the earthquakes which had the highest casualties from the last 30 years in Iran, Rudbar Manjil earthquakes in Gilan province, a Bam earthquake in Kerman province, and three recent earthquakes (from 2017-2020) in Kermanshah province should be mentioned. The city of Kermanshah is located in the west of Iran and its area is about 95.97 square $\mathrm{km}$ and its population is approximately $1,100,000$. This research explores a case study related to Kermanshah province. An earthquake measuring 7.3 on the Richter scale shook Kermanshah province in 2017, killing 630 people and injuring more than 8,435. The second most recent earthquake in Kermanshah province in 2018 reported with a magnitude of 6 on the Richter scale, killing three people and leaving 243 injured and the third earthquake was in 2018 with a magnitude of 6.3 on the Richter scale which killed 1 person and left more than 761 persons injured (see [54]). In terms of the prevalence of disabilities, the number of PWDs in the province of Kermanshah is much higher than the average number per state in Iran and this province has 44 disability rehabilitation centers. In total the province of Kermanshah has 37,782 PWDs with 9621 having intellectual, 4646 having a vision, 14247 having physical, 2881 having mental, 6213 having heard, and 174 having spoken disabilities.

According to recent statistics, the frequency of disasters is highly rated in Iran especially in Kermanshah province. Also, studies show that during natural disasters PWDs are being neglected in evacuation which significantly increases the likelihood of causalities and injuries for this group of people. This major inequality gap has motivated us to address their transportation issues by formulating an evacuation network model to transport PWDs whilst considering the number and variety of their disabilities. Finding an effective approach can assist crisis managers in carrying out evacuation operations in affected areas. Some of these people who require help are do not get any assistance from their relatives. Since private data related to PWDs cannot be released due to confidentiality issues, 493 zones in the city of Kermanshah were identified according to where PWDs who potentially need help live. As mentioned before, these places are marked in black on the map. In this section, according to Fig. 5, there are 493 demand points (disabled locations), 6 shelter points (five equipped shelters and one backup 
shelter). These shelters include Imam Khomeini Hospital, Tamin Ejtemai Hospital, Shahid Beheshti High School, Shahed High School, Islamic Azad University of Kermanshah, and Azadi Stadium. Also, the Islamic Azad University of Kermanshah is considered a backup shelter. Vehicles are also dispatched from the depot, which is marked with green in Fig. 5. The distances between all nodes including pick-up, shelter, and depot nodes are saved in a 500×500 matrix. The speed of bus, van, and passenger cars is considered to be $\mathrm{X}, 1.2 \mathrm{X}$, and 1.6X, respectively. For example, if we assume an average bus speed of $30 \mathrm{~km} / \mathrm{h}$, the average speed of a van is $36 \mathrm{~km} / \mathrm{h}$ and then the average passenger car speed is $48 \mathrm{~km} / \mathrm{h}$. 10 buses, $40 \mathrm{vans}$, and 18 cars were considered for the transportation of PWDs. The capacity of buses for transporting PWDs is 20, vans ten and passenger cars four. Then, the case study information was entered in the $(\mu+1) \mathrm{EA}$ and it was run 10 times and the results are displayed in Table 6.

\{Please insert Fig. 5 about here.

\{Please insert Table 6 about here.

As mentioned before, evacuation time is one of the main factors in reducing casualties in natural disasters. Therefore, the objective function of the proposed model is a Min Max function, which minimizes the longest tour time. According to Table 6 after 10 runs, the average time of the longest tour was 54.4 minutes and the standard deviation of the objective function values for 10 runs was 1.01 minutes. These numbers show that the quality of the solutions obtained is acceptable and if there are preparedness plans, this number of demand points can be evacuated in less than an hour. It is worth mentioning that this evacuation plan is intended to be put in place before the occurrence of a disaster. The results show that 68 tours are formed for the transportation of 493 PWDs, which means that 68 vehicles were in use. In total buses carried 100 people, vans carried 330 people and the passenger cars carried 63 people. Of the 68 vehicles, 10 are buses, 40 are vans and 18 are passenger cars where half of all vehicle operators are trained operators, and the other half are ordinary operators. Out of these 68 tours, two tours were formed with 11 nodes, 25 tours were with 10 nodes, four tours with nine nodes, seven tours with eight nodes, four tours with seven nodes, three tours with six nodes, two tours with five nodes and 13 tours formed with four nodes, five tours are formed with three nodes and three tours are formed with two nodes. The bus tour with the most nodes is 11 and the passenger car tour with the least nodes is 2 . One out of 68 tours are displayed below:

$$
1 \rightarrow 441 \rightarrow 474 \rightarrow 472 \rightarrow 476 \rightarrow 99 \rightarrow 81 \rightarrow 75 \rightarrow 160 \rightarrow 221 \rightarrow 137 \rightarrow 245 \rightarrow 498 \rightarrow 1
$$

In the case where the number of vehicles is considered 66 instead of 68 , a comparison between the tours with 68 vehicles and 66 vehicles shows that the capacity of the vehicles is used more in 66 vehicles. The following is a sample of the tours with 66 vehicles. The increased usage of vehicle capacity is true according to the following tour:

$$
\begin{aligned}
& 1 \rightarrow 496 \rightarrow 154 \rightarrow 480 \rightarrow 465 \rightarrow 233 \rightarrow 75 \rightarrow 280 \rightarrow 120 \rightarrow 296 \rightarrow 298 \rightarrow 252 \rightarrow 255 \\
& \rightarrow 276 \rightarrow 294 \rightarrow 59 \rightarrow 68 \rightarrow 496 \rightarrow 1
\end{aligned}
$$


The results of using 68 vehicles compared to 66 vehicles showed that the time of the longest tours in the two cases was 53 minutes and 60.34 minutes, respectively. By using two more vehicles with a capacity of 10 people, the time of the longest tour has been reduced by 7.34 minutes. With 68 vehicles, the maximum number of unoccupied seats on buses is 6 seats, while with 66 vehicles, it is three seats. Therefore, in these conditions, there are two conflicting situations. If the evacuation time is considered as the priority, it is better to allocate more vehicles to shorten the evacuation time, however, the evacuation time increases if the seats are unoccupied. The results of the Kruskal-Wallis statistical test illustrated that opening the sixth shelter (as a backup shelter) did not have a significant effect on reducing the longest time in the evacuation network with a confidence interval of $95 \%$. Since the location of the sixth shelter (Kermanshah Azad University) is on the outskirts of the city and there is a longer travel distance fewer PWDs are transported to this shelter, which is approved by the results of the model (see Fig. 6). The results of the Kruskal-Wallis statistical test showed that by adding five-passenger cars (five-passenger cars have the equivalent capacity to one bus) to the original case (with 68 vehicles), the time of the longest tour in the evacuation network is significantly reduced with a confidence interval of $95 \%$. Therefore, the substantial improvement of evacuation time caused by this type of vehicle prompted crisis managers to put more orders (prepared) for passenger cars in to immensely reduce the network evacuation time in the occurrence of natural disasters. When the size of the initial fleet, a bus vehicle with a capacity of 20 people is added, the longest tour time on the network is 60 minutes. While if we add five vehicles with a capacity of four people (20 people in total) to the evacuation network instead of a single bus, the time of the longest tour is 52.7 minutes. Thus, comparisons show that adding passenger cars can reduce the time of the longest tours by 7.3 minutes.

The statistical analysis also shows that there is a significant difference between the average time of the longest tour in the two cases (adding bus and adding passenger cars with equivalent capacity). Adding a medium capacity vehicle (van) compared to passenger vehicles (equivalent capacity) makes no significant difference between the average time of the longest tour. Therefore, it is recommended to use medium and small capacity vehicles as much as possible for evacuation, to substantially reduce the time of tours. Furthermore, to decrease the longest tour time in the network a sensitivity analysis has been conducted such that an ordinary operator driving a paratransit van (PVWOTO) is switched for a trained operator (PVWTO). The results indicated that if this would occur the longest tour time would decrease by $4.5 \%$. Logically trained operators can provide service to more PWDs as they have appropriate qualifications (PVTWOs are compatible with G1, G2, G3, and G4) meaning that they can access all demand nodes. Conversely, ordinary operators have restrictions on the demand nodes they can visit and the disabilities they are compatible with since they do not have the required qualifications (PVTOWOs are only compatible with G1 and G2), and because of this, these operators are forced to not visit closer nodes and leave those PWDs, and instead go to more distant nodes. Moreover, this inconvenience results in those PWDs being picked up by distant PVTWOs which would increase the evacuation time and the risk for those left PWDs during an emergency. By PVTWOs traveling to left PWDs, the waiting time for other PWDs in its route increases and this disrupts the consistency and efficiency of transportation in the evacuation network while also increasing the risk for PWDs waiting to be picked up. Ultimately the lack 
of skill of operators immensely contributes to increasing evacuation time and increased risk of the injury and fatality of PWDs during disasters.

\{Please insert Fig. 6 about here.\}

\section{Conclusion}

Firstly, in this study, the transportation needs of PWDs in the context of the occurrence of natural hazard emergencies were addressed. One of the most important problems is correctly identifying and communicating with PWDs when evacuating and transporting them during the occurrence of natural disasters. PWDs, their families, and organizations that support PWDs face major challenges during natural disasters including PWDs having a lack of equal access to appropriate facilities and resources in communities with ordinary people, special needs of PWDs and complexity of their health conditions, lack of shelters with appropriate housing standards and insufficiency in trained and experienced people to help PWDs.

In this paper, an MILP model has been proposed for evacuation and transportation of PWDs to shelters considering features such as the variety of types of disabilities, the heterogeneity of the types of vehicles for increased safety and speed of action in crisis situations, and the compatibility of the type of disability with the type of vehicle. These features and the allocation of experienced operators and ordinary operators are considered for dispatching vehicles to the requested location. To ensure fairness during the evacuation and transfer of PWDs to shelters, the min-max objective function was utilized. This objective function balances the evacuation time for the tours that are formed. To solve the model on a large scale, the $(\mu+1)$ EA was designed, and 27 instances were generated randomly and solved with the $(\mu+1)$ EA algorithm. The results verify the performance of the $(\mu+1)$ EA. Also, a case study was implemented in the city of Kermanshah. Furthermore, three types of sensitivity analysis were performed about the case study. The first analysis illustrated that adding small-sized vehicles (passenger cars) and medium-sized vehicles (vans) with equivalent capacity instead of buses significantly reduces evacuation time (tours time).

The results of the analysis also demonstrated that adding a backup shelter does not have a significant effect on reducing evacuation time. The reason for its insignificance is that adding the backup shelter does not affect the longest tour (the time of the longest tour) since the location of this backup shelter is located in the outskirts of the city, and as a result, few PWDs are allocated to this shelter. The results also showed that adding only two medium-sized vehicles (vans) reduces the evacuation time of the longest tour (the longest tour time) to 7.5 minutes. About the managerial implications, it can be stated that in the occurrence of natural disasters, it is beneficial to use more vans or cars to increase the speed of evacuation tours. Therefore, the crisis managers of organizations should focus on medium and small-sized vehicles to reduce evacuation time and indirectly reduce the risk of people being trapped during natural disasters (due to delays in evacuation). Moreover, the location of shelters should also be considered as it plays an important role in reducing evacuation time. Therefore, emergency planners should have predetermined scenarios for allocation of shelters to prevent deaths and injuries of PWDs in affected areas, so that during the occurrence of an accident, based on the scenario that takes priority, more effective shelters can be opened to prevent wastage of resources in critical 
situations. For future research, the uncertainty of demand points and unavailability of some links in the network (arcs) due to disruptions should be considered. This disturbance results in slow movement between paths and longer evacuation times, which increases the risk of people involved in the disaster.

\section{References}

[1] Christie, N., Griffin, L., Chan, N., Twigg, J., and Titheridge, H. "Private needs, public responses: vulnerable people's flood-disrupted mobility", Disaster Prevention and Management, 25(2), pp. 244-260 (2016).

[2] Marsh, T.J., and Hannaford, J., "The summer 2007 floods in England and Wales - A hydrological appraisal", Centre for Ecology and Hydrology, (2008).

[3] Pitt, M. "The Pitt Review: Lessons from the 2007 Floods", The Cabinet Office, London, (2008).

[4] Jinwoo, L., Zheng, Z., Kashfi, S., Chia, J., and Yi, R. "Observation of Bus Ridership in the Aftermath of the 2011 Floods in Southeast Queensland, Australia”, In: Proceedings of the $9^{\text {th }}$ Annual International Conference of the International Institute for Infrastructure Renewal and Reconstruction, Brisbane, Australia, 8-10 July 2013.

[5] Wolshon, B. "Planning for the evacuation of New Orleans", Institute of Transportation Engineers, 72(2), pp. 44-49 (2002).

[6] Walker, G., and Burningham, K. "Flood risk, vulnerability and environmental justice: Evidence and evaluation of inequality in a UK context", Critical Social Policy, 31(2), pp. 216-240 (2011).

[7] Thywissen, K. "Components of risk: A comparative glossary", United Nations University, Institute for Environment and Human Security, Source/2, Publication of UNU -EHS (2006).

[8] Alexander, D., Gaillard, J.C., and Wisner, B. "Disability and disaster", In: B. Wisner, J.C. Gaillard and I. Kelman, Handbook of Hazards and Disaster Risk Reduction, pp. 413-423 (2012).

[9] Hans, A., Patel, A.M., Sharma, R.K., Prasad, D., Mahapatra, K., and Mohanty, R. "Mainstreaming disability in disaster management: A tool kit", New Delhi: United Nations Development Program, (2008).

[10] https://www.ifrc.org/PageFiles/99876/WDR2007-English.pdf IFRC (International Federation of Red Cross and Red Crescent Societies), World disaster report: Focus on discrimination. Geneva: IFRC, (2007).

[11] Strough, L.M., and Kang, D. "The Sendai framework for disaster risk reduction and persons with disabilities", International Journal of Disaster Risk Science, 6, pp.140-149 (2015).

[12] Apte, A., Heath S., Pico, A. and Tan, Y.H.R. "Evacuating people with mobility-challenges in a short-notice disaster", Decision Sciences Institute, 46(4), pp. 731-754 (2015).

[13] https://www.ncbi.nlm.nih.gov/books/NBK11420/

[14] Apte, A., and Heath, S. "Request and response processes for Department of Defense support during domestic disasters", Journal of Homeland Security and Emergency Management, 8(1), (2011).

[15] Nieburg, P., Waldman, R.J., and Krumm, D.M. "Evacuated populations - Lessons from foreign refugee crises", The New England Journal of Medicine, 353(15), pp. 1547-1549 (2005).

[16] Litman, T. "Lessons from Katrina and Rita: What Major Disasters Can Teach Transportation Planners", Journal of Transportation Engineering, 132(1), pp. 11-18 (2006).

[17] Malpass, A., West, C., Quaill, J., and Barker, R. "Experiences of individuals with disabilities sheltering during natural disabilities: An integrative review", Australian Journal of Emergency Management, 34(2), pp. 60-65(2019). 
[18] Mace, S.E., Doyle, C. J, Askew, K., Bradin, S., Baker, M., Joseph, M.M., and Sorrentino, A. "Planning - considerations for persons with access and functional needs in a disaster-Part 1: Overview and legal", American Journal of Disaster Medicine, 13(2), pp. 69-83(2018).

[19] www.ons.gov.uk/peoplepopulationandcommunity

[20] Wong, S., Broader, J., and Shaheen, S. "A Checklist of Immediate Actions for Addressing COVID-19 as Part of Evacuation Planning". UC Office of the President: University of California Institute of Transportation Studies. http://dx.doi.org/10.7922/G25H7DJT Retrieved from https://escholarship.org/uc/item/6311c0jx(2020).

[21] Detti, P., Papalini, F., and Lara, G.Z.M.D. "A multi-depot dial-a-ride problem with heterogeneous vehicles and compatibility constraints in healthcare”, Omega, 70, pp. 1-14 (2017).

[22] Parragh, S.N., Doerner, K.F., and Hartl, R.F. "Variable neighborhood search for the dial-a-ride problem", Computers and Operations Research, 37(6), pp. 1129-1138 (2010).

[23] Melachrinoudis, E., Ilhan, A.B., and Min, H. "A dial-a-ride problem for client transportation in a health-care organization", Computers and Operations Research, 34(3), pp. 742-759 (2007).

[24] Parragh, S.N., Cordeau, J.-F., Doerner, K.F., and Hartl, R.F. "Models and algorithms for the heterogeneous dial-a-ride problem with driver-related constraints", Operations Research Spectrum, 34(3), pp. 593-633 (2012).

[25] Lehuédé, F., Masson, R., Parragh, S.N., Péton, O., and Tricoire, F. "A multi-criteria large neighbourhood search for the transportation of disabled people", Journal of the Operational Research Society, 65(7), pp. 983-1000 (2014).

[26] Rais, A., Alvelos, F., and Carvalho, M.S. "New mixed integer-programming model for the pickup and delivery problem with transshipment", European Journal of Operational Research, 235(3), pp. 530-539 (2014).

[27] Feng, L., Vodopivec, N., and Miller-Hooks, E. "Supporting mobility-impaired populations in emergency evacuations", Transportation Research Record, 2532(1), pp. 118-128 (2015).

[28] Faria, A.D., Yamashita, M., Tozi, L.A., Souza, V.J.D., and Junior, I.D.B. "Dial-a-ride Routing System: the study of mathematical approaches used in public transport of people with physical disabilities", Proceedings of the $12^{\text {th }}$ World Conference on Transport Research (WCTR) Society, Lisbon, Portugal, 11-15 July 2010.

[29] Melachrinoudis, E., and Min, H. "A tabu search heuristic for solving the multi-depot, multivehicle, double request dial-a-ride problem faced by a healthcare organisation", International Journal of Operational Research, 10(2), pp. 214-239 (2011).

[30] Reinhardt, L.B., Clausen, T., and Pisinger, D. "Synchronized dial-a-ride transportation of disabled passengers at airports", European Journal of Operational Research, 225(1), pp. 106-117 (2013).

[31] Parragh, S.N. "Introducing heterogeneous users and vehicles into models and algorithms for the dial-a-ride problem", Transportation Research Part C: Emerging Technologies, 19(5), pp. 912930 (2011).

[32] Riedler, M., and Raidl, G. "Solving a selective dial-a-ride problem with logic-based Benders decomposition", Computers and Operations Research, 96, pp. 30-54 (2018).

[33] Cordeau, J.-F., and Laporte, G. "A tabu search heuristic for the static multi-vehicle dial-a-ride problem”, Transportation Research Part B: Methodological, 37(6), pp. 579-594 (2003).

[34] Parragh, S.N., Doerner, K.F., Hartl, R.F. and Gandibleux, X. "A Heuristic Two-Phase Solution Approach for the Multi-Objective Dial-A-Ride Problem”, Networks, 54(4), pp. 227-242 (2009).

[35] Molenbruch, Y., Braekers, K., Caris, A., and Berghe, G.V. "Multi-directional local search for a bi-objective dial-a-ride problem in patient transportation", Computers and Operations Research, 77, pp. 58-71 (2017). 
[36] Cheng, C.B. "A location based dial-a-ride service system for the elderly and the disabled in Taiwan", In: Proceedings of the Fourth International Conference on Ubi-Media Computing, Sao Paulo, Brazil, 3-4 July 2011, pp. 140-145 (2011).

[37] Tellez, O., Vercraene, S., Lehuédé, F., Péton, O., and Monteiro, T. "Dial-a-ride problem for disabled people using vehicles with reconfigurable capacity", In: Proceedings of the $20^{\text {th }}$ IFAC World Congress of the International Federation of Automatic Control (IFAC), Toulouse, France, 9-14 July 2017.

[38] Ropke, S., and Pisinger, D. "An adaptive large neighborhood search heuristic for the pickup and delivery problem with time windows", Transportation Science, 40(4), pp. 455-472 (2006).

[39] Kaisar, E.L., Hess, L. and Palomo, A.B.P. "An emergency evacuation planning model for special needs populations using public transit systems", Journal of Public Transportation, 15(2), pp. 4569 (2012).

[40] Dhamala, T.N., and Adhikari, I.M. "On Evacuation Planning Optimization Problems from Transit-based Perspective", International Journal of Operations Research, 15(1), pp. 29-47 (2018).

[41] Murray-Tuite, P. and Wolshon, B. "Evacuation transportation modeling: an overview of research, development, and practice", Transportation Research Part C, 27, pp. 25-45 (2013).

[42] Bian, R., and Wilmot, C.G. "An analysis on transit pick-up points for vulnerable people during hurricane evacuation: A case study of New Orleans", International Journal of Disaster Risk Reduction, 31, pp. 1143-1151 (2018).

[43] Bayram, V. "Optimization models for large scale network evacuation planning and management: A literature review", Surveys in Operations Research and Management Science, 21(2), pp. 63-84 (2016).

[44] Amideo, A.E. , Scaparra, M.P., and Kotiadis. K. "Optimising shelter location and evacuation routing operations: The critical issues", European Journal of Operational Research, 279, pp. 279295 (2019).

[45] Shahparvari, S., and Abbasi, B. "Robust stochastic vehicle routing and scheduling for bushfire emergency evacuation: An Australian case study", Transportation Research Part A: Policy and Practice, 104, pp. 32- 49 (2017).

[46] Dulebenets, M.A., Pasha, J., Kavoosi, M., Abioye, O.F., Ozguven, E.E., Moses, R., Boot, W.R., and Sando, T. "Multiobjective optimization model for emergency evacuation planning in geographical locations with vulnerable population groups", Journal of Management in Engineering, 36(2), pp. 1-17 (2020).

[47] Baou, E. Koutras, V.P., Zeimpekis, V., and Minis, I. "Emergency evacuation planning in natural disasters under diverse population and fleet characteristics", Journal of Humanitarian Logistics and Supply Chain Management, 8(4), pp. 447-476 (2018).

[48] Flores, I., Ortuño, M.T., and Tirado, G., and Vitoriano, B. "Supported Evacuation for Disaster Relief through Lexicographic Goal Programming”, Mathematics, 8(4), pp. 2-20 (2020).

[49] Zhao, X., Xu, W., Ma, Y., Qin, L., Zhang, J., and Wang, Y. "Relationships between evacuation population size, earthquake emergency shelter capacity, and evacuation time", International Journal of Disaster Risk Science, 8, pp. 457-470 (2017).

[50] Bolia, N.B. "Operating strategies of buses for mass evacuation", Safety Science, 111, pp. 167178 (2019).

[51] Swamy, R., Kang, J.E., Batta, R., and Chung, Y. "Hurricane evacuation planning using public transportation", Socio-Economic Planning Sciences, 59, pp. 43-55 (2017).

[52] Dulebenets, M.A., Pasha, J., Abioye, O.F., Kavoosi, M., Ozguven, E.E., Moses, R., Boot, W.R., and Sando, T. "Exact and heuristic solution algorithms for efficient emergency evacuation in areas 
with vulnerable populations", International Journal of Disaster Risk Reduction, 39, pp. 1-18 (2019).

[53] Gao, X., Nayeem , M.K., and Hezam, I.M. "A robust two-stage transit-based evacuation model for large-scale disaster response”, Measurement, 145, pp. 713-723 (2019).

https://ourworldindata.org/natural-disasters

[54] https://en.wikipedia.org/wiki/List_of_earthquakes_in_Iran

Sadoullah Ebrahimnejad is an Associate Professor of Industrial Engineering at Islamic Azad University - Karaj Branch in Iran. He received his B.Sc. degree from Iran University of Science and Technology in 1986, M.Sc. degree from Amirkabir University of Technology in 1993, and Ph.D. degree from Islamic Azad University Science and Research Branch in Tehran in 2001. His research interests are Fuzzy MADM/MODM, SCM, Operations management, Optimization models, Risk management, Multi-criteria network optimization. He published more than 200 papers in reputable academic journals and conferences.

Michelle Villeneuve is an Associate Professor at The University of Sydney and leads the disability-inclusive community development work stream at the Centre for Disability Research and Policy. Michelle's international program of research addresses inequities that people with disability experience in everyday living and situations of natural hazard disaster, and other emergencies. Her research informs both conceptual and practical dimensions of inter-professional collaboration and resource utilization. She brings to this research over 20 years of experience working in regions of conflict and natural hazard disaster to develop community-led programs and services, and re-build opportunities for people with disability. She published more than 200 journal/conference papers and reports.

Reza Tavakkoli-Moghaddam is a Professor of Industrial Engineering at the College of Engineering, University of Tehran, Iran. He obtained his Ph.D., M.Sc. and B.Sc. degrees in Industrial Engineering from the Swinburne University of Technology in Melbourne (1998), the University of Melbourne in Melbourne (1994), and the Iran University of Science and Technology in Tehran (1989), respectively. He serves as the Editor-in-Chief of the Journal of Industrial Engineering published by the University of Tehran and as the Editorial Board member of nine reputable academic journals. He is the recipient of the 2009 and 2011 Distinguished Researcher Awards and the 2010 and 2014 Distinguished Applied Research Awards at the University of Tehran, Iran. He has been selected as the National Iranian Distinguished Researcher in 2008 and 2010 by the MSRT (Ministry of Science, Research, and Technology) in Iran. He has obtained an outstanding rank as the top 1\% scientist and researcher in the world elite group since 2014. He also received the Order of Academic Palms Award as a distinguished educator and scholar for the insignia of Chevalier dans l'Ordre des Palmes Academiques by the Ministry of National Education of France in 2019. He has published 5 books, 32 book chapters, and more than 1000 journal and conference papers. 


\section{Tables}

Table 1. Six modes for vehicle-operator and four disability groups

Table 2. Comparison of the CPLEX solver and $(\mu+1)$ EA

Table 3. Allocation pickup nodes to vehicles in the exact method and $(\mu+1) \mathrm{EA}$

Table 4. Statistical analysis

Table 5. The effect of restart mechanism on the quality of solutions

Table 6. Obtained solutions in ten runs

\section{Figures}

Fig. 1. Paratransit vehicles with different configurations.

Fig. 2. Chromosome representation (V: Type of vehicle, D: Depot node, P: Pickup node, S: Shelter node)

Fig. 3. Presentation of the mutation operator

Fig. 4. Comparison of run times

Fig. 5. Kermanshah (Bakhtaran) map

Fig. 6. Map (Source: Google Maps) 
Tables

Table 1. Six modes for vehicle-operator and four disability groups

\begin{tabular}{|c|c|c|c|c|c|c|}
\hline $\begin{array}{c}\text { Type of } \\
\text { Disability }\end{array}$ & PVWTO & BWOTO & BWTO & CWOTO & CWTO & PVWOTO \\
\hline Mode & 3 & 2 & 1 & 6 & 5 & 4 \\
\hline G1 & 1 & 1 & 1 & 1 & 1 & 1 \\
\hline G2 & 1 & 0 & 0 & 0 & 0 & 1 \\
\hline G3 & 1 & 0 & 1 & 0 & 1 & 0 \\
\hline G4 & 1 & 0 & 0 & 0 & 0 & 0 \\
\hline
\end{tabular}

BWTO: Bus with a trained operator, BWOTO: Bus without a trained operator, PVWTO: Paratransit vehicle with a trained operator, PVWOTO: Paratransit vehicle without a trained operator, CWTO: Car with trained operator, CWOTO: Car without, trained operator.

Table 2. Comparison of the CPLEX solver and $(\mu+1)$ EA

\begin{tabular}{|c|c|c|c|c|c|c|c|c|c|c|}
\hline & & & PLEX & ver & & & $(\mu+1) \mathrm{E}$ & & & \\
\hline 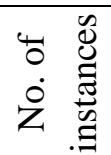 & $\begin{array}{c}\text { Problem size } \\
(P \times S \times V \times D)\end{array}$ & $\begin{array}{l}\text { OF } \\
(\mathrm{min})\end{array}$ & $\begin{array}{c}\text { Status } \\
\text { OPS } \\
\text { /NOPS }\end{array}$ & $\begin{array}{l}\text { CPU } \\
\text { time } \\
(\min )\end{array}$ & $\begin{array}{c}\text { Mean } \\
\text { OF } \\
(\mathrm{min})\end{array}$ & $\begin{array}{c}\text { Best } \\
\text { solution }\end{array}$ & $\begin{array}{c}\text { Status } \\
\text { OPS } \\
\text { /NOPS }\end{array}$ & $\begin{array}{l}\text { Standard } \\
\text { deviation }\end{array}$ & $\begin{array}{l}\text { CPU } \\
\text { time } \\
\text { (sec.) }\end{array}$ & OF gap (\%) \\
\hline 1 & $15 \times 4 \times 3 \times 1$ & 192 & OPS & 57.92 & 192.66 & 192 & OPS & 0.544 & 143.44 & 0 \\
\hline 2 & $20 \times 4 \times 3 \times 1$ & 255 & OPS & 413.33 & 255.23 & 255 & OPS & 0.737 & 172.9 & 0 \\
\hline 3 & $25 \times 4 \times 3 \times 1$ & 385 & OPS & 13.43 & 358.3 & 385 & OPS & 0.674 & 178.77 & 0 \\
\hline 4 & $30 \times 4 \times 3 \times 2$ & 531 & OPS & 3.70 & 532.8 & 531 & OPS & 1.549 & 214.14 & 0 \\
\hline 5 & $50 \times 4 \times 6 \times 2$ & 376 & OPS & 5073.32 & 384.9 & 380 & NOPS & 3.21 & 327.27 & $-0.01(-1)$ \\
\hline 6 & $75 \times 4 \times 8 \times 2$ & 501 & NOPS & 7201.85 & 467.6 & 465 & NOPS & 1.95 & 354.176 & $0.07(7)$ \\
\hline 7 & $90 \times 6 \times 10 \times 3$ & N/A & N/A & 7200.41 & 472.6 & 466 & NOPS & 3.86 & 450.994 & N/A \\
\hline 8 & $110 \times 5 \times 11 \times 3$ & N/A & N/A & 7203.67 & 498.4 & 493 & NOPS & 4.32 & 608.399 & N/A \\
\hline 9 & $150 \times 6 \times 16 \times 3$ & N/A & N/A & 7203.67 & 507.7 & 498 & NOPS & 5.37 & 641.31 & N/A \\
\hline 10 & $15 \times 4 \times 3 \times 1$ & 266 & OPS & 620.87 & 266 & 266 & OPS & 0 & 142.477 & 0 \\
\hline 11 & $20 \times 4 \times 3 \times 1$ & 359.3 & OPS & 7200.13 & 360.1 & 359.3 & OPS & 0.421 & 167.8 & 0 \\
\hline 12 & $25 \times 4 \times 3 \times 1$ & 545 & OPS & 5.26 & 545.2 & 545 & OPS & 0.421 & 162.63 & 0 \\
\hline 14 & $50 \times 4 \times 6 \times 2$ & 540 & OPS & 1463.59 & 548.2 & 546 & NOPS & 1.31 & 280.3 & $-0.01(-1)$ \\
\hline 15 & $75 \times 4 \times 8 \times 2$ & 686 & NOPS & 7200.82 & 660.9 & 656 & NOPS & 2.92 & 358.4 & $0.044(4.4)$ \\
\hline 16 & $90 \times 6 \times 10 \times 3$ & 724 & NOPS & 7200.91 & 660.4 & 652 & NOPS & 3.92 & 462.7 & $0.099(9.9)$ \\
\hline 17 & $110 \times 5 \times 11 \times 3$ & N/A & N/A & 7200.91 & 707.3 & 701 & NOPS & 6.50 & 459.4 & N/A \\
\hline 18 & $150 \times 6 \times 16 \times 3$ & N/A & N/A & 7202.32 & 708.1 & 700 & NOPS & 6.51 & 584 & N/A \\
\hline 19 & $15 \times 4 \times 3 \times 1$ & 29 & OPS & 15.18 & 29.2 & 29 & OPS & 0.322 & 169.2 & 0 \\
\hline 20 & $20 \times 4 \times 3 \times 1$ & 39.3 & OPS & 2.73 & 40.3 & 39.3 & OPS & 0.974 & 204.1 & 0 \\
\hline
\end{tabular}




\begin{tabular}{|c|c|c|c|c|c|c|c|c|c|c|}
\hline 21 & $25 \times 4 \times 3 \times 1$ & 66 & OPS & 2.26 & 66 & 66 & OPS & 0 & 174.5 & 0 \\
\hline 22 & $30 \times 4 \times 3 \times 2$ & 82 & OPS & 4.63 & 82.4 & 82 & OPS & 0.515 & 187.6 & 0 \\
\hline 23 & $50 \times 4 \times 6 \times 2$ & 69 & NOPS & 7203.24 & 67.9 & 66 & NOPS & 1.79 & 174.5 & $0.043(4.3)$ \\
\hline 24 & $75 \times 4 \times 8 \times 2$ & 77.3 & NOPS & 7200.538 & 64.5 & 60 & NOPS & 3.37 & 363.9 & $0.22(22)$ \\
\hline 25 & $90 \times 6 \times 10 \times 3$ & 113 & NOPS & 7200.944 & 75.8 & 72 & NOPS & 2.52 & 346.3 & $0.36(36)$ \\
\hline 26 & $110 \times 5 \times 11 \times 3$ & 119 & NOPS & 7201.489 & 81.7 & 78 & NOPS & 3.26 & 550.3 & $0.344(34.4)$ \\
\hline 27 & $150 \times 6 \times 16 \times 3$ & N/A & N/A & 7201.848 & 79.1 & 74 & NOPS & 4.23 & 790.5 & N/A \\
\hline
\end{tabular}

P: Pickup nodes, S: shelter nodes, V: vehicle, D: Depot, OF: objective function, OPS: Optimal Solution, NOPS: Not Optimal Solution, N/A: After two hours did not reach to feasible solution.

Table 3. Allocation pickup nodes to vehicles in the exact method and $(\mu+1) \mathrm{EA}$

\begin{tabular}{|c|l|l|}
\hline & \multicolumn{1}{|c|}{ Exact method by CPLEX solver } & \multicolumn{1}{c|}{$(\mu+1)$ EA } \\
\hline V1 & $1 \rightarrow 9 \rightarrow 13 \rightarrow 11 \rightarrow 5 \rightarrow 4 \rightarrow 12 \rightarrow 2 \rightarrow 8 \rightarrow 15 \rightarrow 14 \rightarrow 28 \rightarrow 1$ & $1 \rightarrow 9 \rightarrow 13 \rightarrow 11 \rightarrow 5 \rightarrow 4 \rightarrow 12 \rightarrow 2 \rightarrow 8 \rightarrow 15 \rightarrow 14 \rightarrow 28 \rightarrow 1$ \\
\hline V2 & $1 \rightarrow 23 \rightarrow 22 \rightarrow 20 \rightarrow 19 \rightarrow 24 \rightarrow 17 \rightarrow 26 \rightarrow 21 \rightarrow 25 \rightarrow 18 \rightarrow 27 \rightarrow 1$ & $1 \rightarrow 19 \rightarrow 20 \rightarrow 21 \rightarrow 24 \rightarrow 23 \rightarrow 22 \rightarrow 3 \rightarrow 25 \rightarrow 18 \rightarrow 26 \rightarrow 28$ \\
\hline V3 & $1 \rightarrow 3 \rightarrow 7 \rightarrow 6 \rightarrow 10 \rightarrow 16 \rightarrow 28 \rightarrow 1$ & $1 \rightarrow 17 \rightarrow 7 \rightarrow 6 \rightarrow 16 \rightarrow 10 \rightarrow 28 \rightarrow 1$ \\
\hline
\end{tabular}

Table 4. Statistical analysis

\begin{tabular}{|l|l|l|l|}
\hline & Mean (min.) & Standard deviation $(\mathrm{Sd})$. & The best solution \\
\hline Original case & 64.31 & 2.7138 & 60.34 \\
\hline Case 1 & 63.67 & 3.5457 & 59.51 \\
\hline Case V1 (mode 1) & 62.04 & 0.9805 & 60.61 \\
\hline Case V2 (mode 2) & 62.48 & 4.59 & 59.7 \\
\hline Case V3 (mode 3) & 60.7 & 2.12 & 58.61 \\
\hline Case V4 (mode 4) & 61.2 & 2.59 & 58.6 \\
\hline Case V5 (mode 5) & 59.36 & 2.44 & 57.24 \\
\hline Case V6 (mode 6) & 60.86 & 1.28 & 59.84 \\
\hline
\end{tabular}

Table 5. Effect of restart mechanism on the quality of solutions

\begin{tabular}{|l|l|}
\hline Operator & Evacuation time (min.) \\
\hline With restart mechanism & 656.13 \\
\hline Without restart mechanism & 756.98 \\
\hline Standard error of mean (SEM) & 20.87 \\
\hline
\end{tabular}

Table 6. Obtained solutions in ten runs

\begin{tabular}{|c|c|c|c|c|c|c|c|c|c|c|c|c|}
\hline No. of runs & 1 & 2 & 3 & 4 & 5 & 6 & 7 & 8 & 9 & 10 & & \\
\hline $\begin{array}{l}\text { OF: } \\
\text { traveled } \\
\text { distance }(\mathrm{m})\end{array}$ & 27215 & 27398 & 26530 & 27408 & 26668 & 27781 & 26953 & 27559 & 26571 & 27951 & $\begin{array}{c}\mu=27203.4, \\
S d=505.7\end{array}$ & \multirow[t]{3}{*}{$\begin{array}{l}\text { With } 68 \\
\text { vehicles }\end{array}$} \\
\hline $\begin{array}{l}\text { Traveled } \\
\text { time (min) }\end{array}$ & 54.43 & 54.796 & 53.06 & 54.816 & 53.336 & 55.562 & 53.906 & 55.118 & 53.142 & 55.902 & $\begin{aligned} \mu & =54.4, \\
S d & =1.0114\end{aligned}$ & \\
\hline $\begin{array}{l}\text { CPU time } \\
(\mathrm{min})\end{array}$ & 163.076 & 225.13 & 343.3 & 128.076 & 167.316 & 125.768 & 441.66 & 156.88 & 238.3 & 134.51 & & \\
\hline $\begin{array}{l}\text { Objective } \\
\text { function }\end{array}$ & 30170 & 32004 & 33582 & 31736 & 31787 & 31667 & 32166 & 32254 & 32213 & 32722 & $\begin{array}{c}\mu=32030.1 \\
S d=864.5\end{array}$ & $\begin{array}{l}\text { With } 66 \\
\text { vehicles }\end{array}$ \\
\hline
\end{tabular}




\begin{tabular}{|l|l|l|l|l|l|l|l|l|l|l|l|}
\hline $\begin{array}{l}\text { traveled } \\
\text { distance } \\
(\mathrm{m})\end{array}$ & & & & & & & & & & & \\
\hline
\end{tabular}

Figures

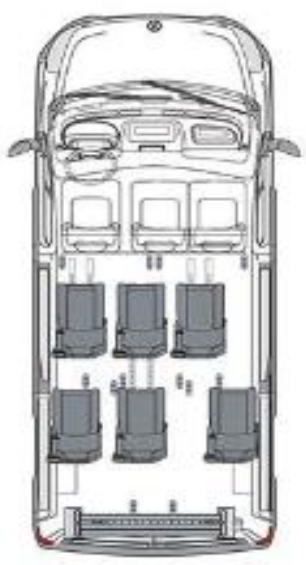

9 田

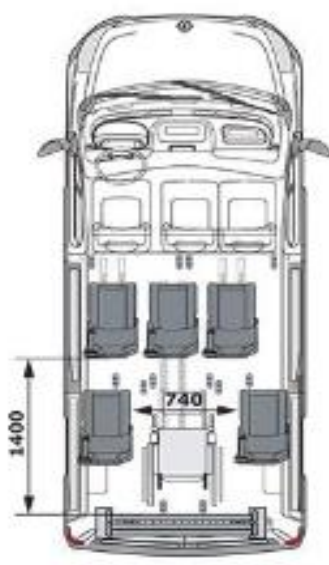

8 붕 +1 웅

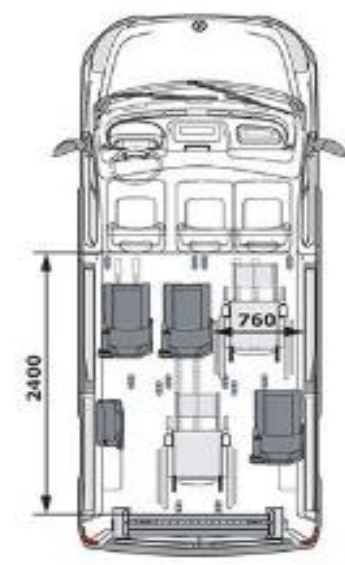

$6[0+2$ 圆

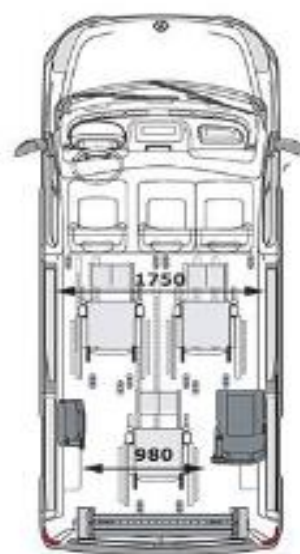

4 团 +3 圆

Fig. 1. Paratransit vehicles with different configurations.

\begin{tabular}{|l|l|l|l|l|l|l|l|l|l|l|l|l|l|l|l|l|l|l|l|l|l|}
\hline & \multicolumn{5}{|c|}{} & & \multicolumn{5}{|c|}{ V1 } & & \multicolumn{7}{|c|}{ V3 } \\
\hline $\mathrm{D}$ & $\mathrm{P}$ & $\mathrm{P}$ & $\mathrm{P}$ & $\mathrm{P}$ & $\mathrm{S}$ & $\mathrm{D}$ & 0 & $\mathrm{D}$ & $\mathrm{P}$ & $\mathrm{P}$ & $\mathrm{S}$ & $\mathrm{D}$ & 0 & $\mathrm{D}$ & $\mathrm{P}$ & $\mathrm{P}$ & $\mathrm{P}$ & $\mathrm{P}$ & $\mathrm{P}$ & $\mathrm{S}$ & $\mathrm{D}$ \\
\hline
\end{tabular}

Fig. 2. Chromosome representation (V: Type of vehicles, D: Depot node, P: Pickup node, S: Shelter node)

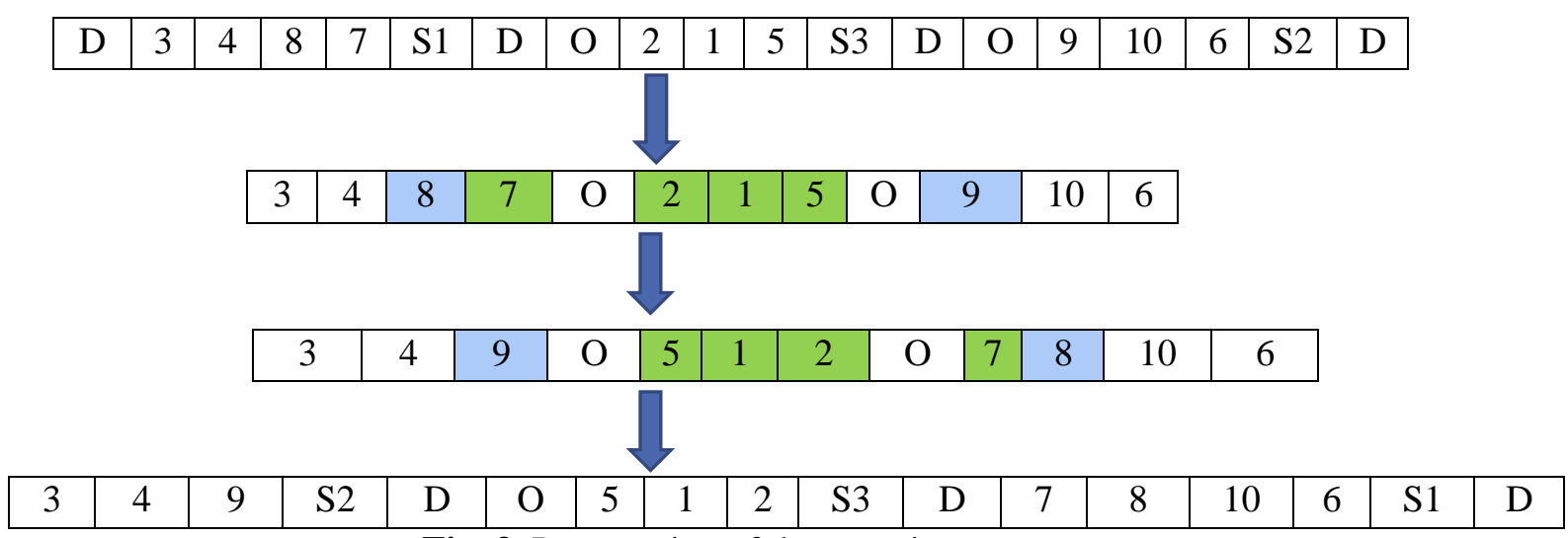

Fig. 3. Presentation of the mutation operator 


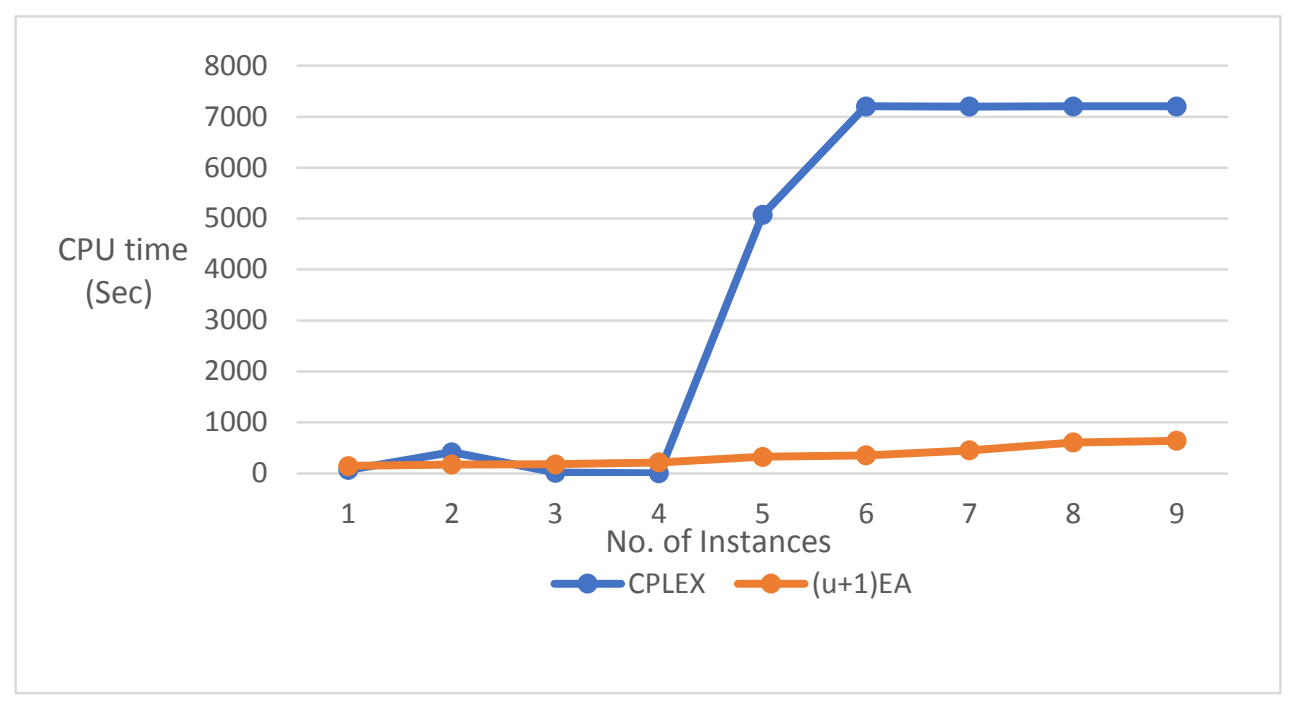

Fig. 4. Comparison of run times

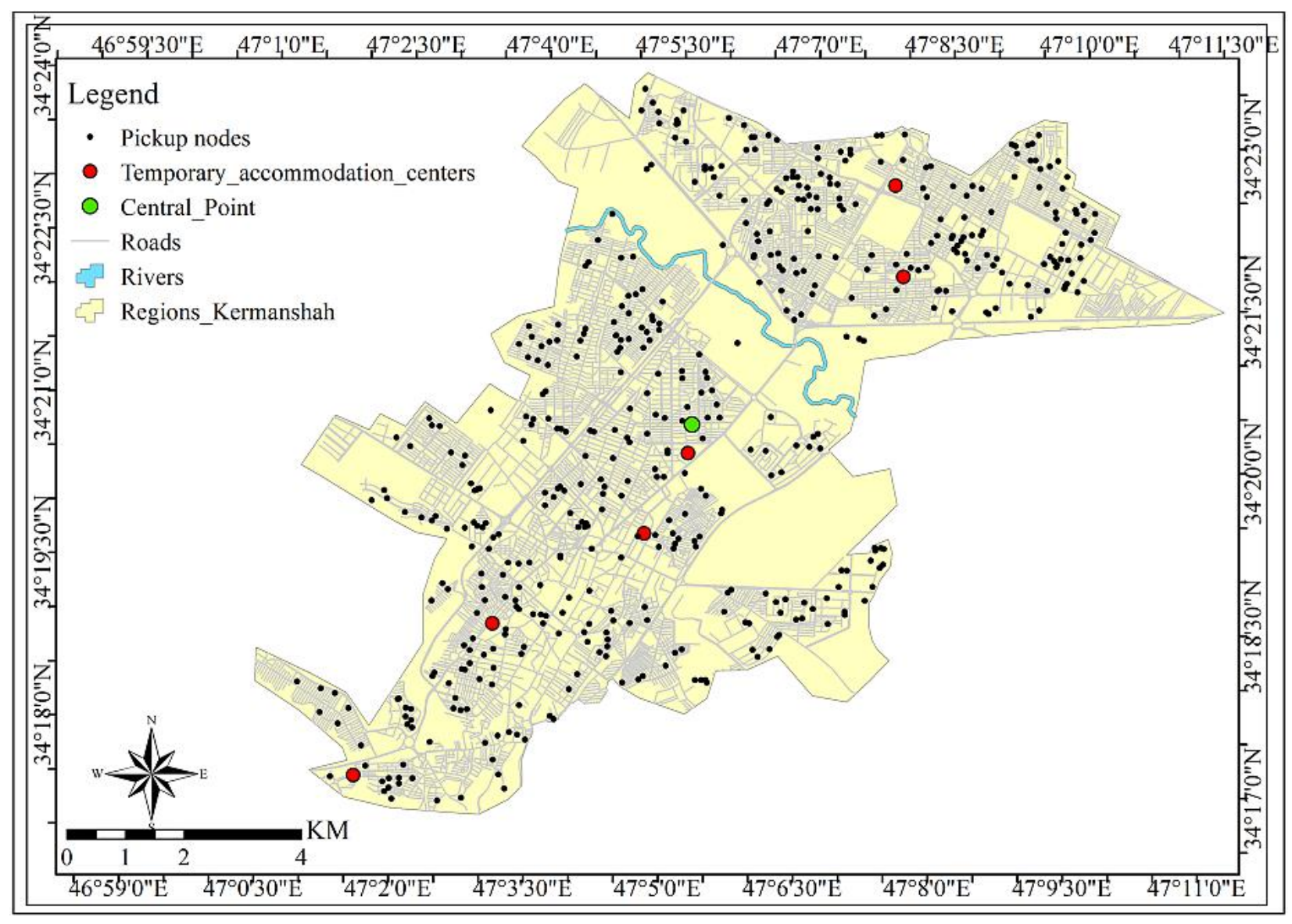

Fig. 5. Kermanshah (Bakhtaran) map 


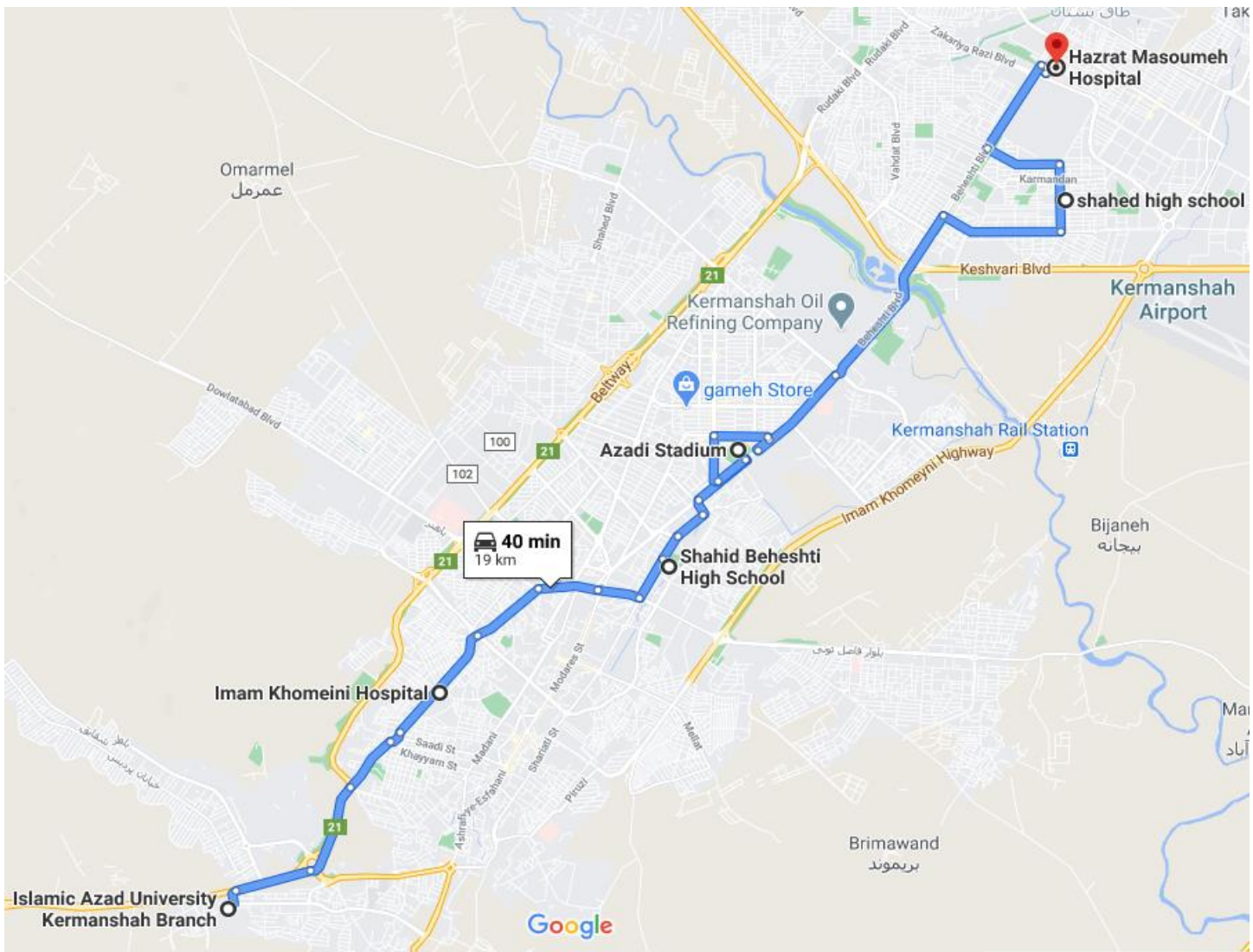

Fig. 6. Map (Source: Google Maps) 